\title{
Theoretical Research on Concentric Circular Selection of Sample Points for Flatness Error Measurement of Revolving Body End-face
}

\author{
Lei Zhang*, Wenying Wang, Peng Guan and Jun Cheng \\ Mechanical Engineering and Automation School, Northeastern University, Shenyang 110004, P. R. China \\ *Corresponding author
}

\begin{abstract}
According to flatness error measurement of revolving body end-face, selection of sample points for flatness error measurement and flatness error evaluation method are mainly researched, the influencing factors are analyzed. Former concentric circular selection of sample points gets many restrictions, the measurement point arranges normally on the straight line that diffused. In this paper, a new concentric circular selection of sample points for flatness error measurement is presented and described by mathematical formulas in detail. The new method of arranging sample points can install quantity and the location of sample points wantonly on circumference direction and diameter direction, and satisfies the needs of the measure of flatness error. Based on using the research methods of relative subjects of other countries for reference, the simulation method for flatness error evaluation of concentric circular selection of sample points is presented, the mathematical model is simulated, and the simulation results are compared and analyzed. Since concentric circular selection of sample points is arranged the central symmetry of sample points, the orthogonal least-square mathematical models of flatness error evaluation for revolving body end-face are built, not only calculation is simple , and can raise the precision of calculation.
\end{abstract}

Keywords-flatness error of revolving body end-face; concentric circular selection of sample points; mathematical description; least-square method

\section{INTRODUCTION}

To a great extent, the method of arranging sample points for flatness error measurement is affected by the method of measurement, evaluation method, the geometric shape of measured workpieces and other factors ${ }^{[1-4]}$. For flatness error measurement of revolving body end-face, using concentric circular to arrange sample points has been narrated in related books. But this method which is still based on gradienter and autocollimation make use of pitch to the measure. Data obtained by the use of indirect measurement estimate flatness error. In the process of arranging sample points, these points that consist of concentric circles cover linear according to radiation patterns. Not only that, this method, which comes from practice and does not do the theoretical research, can't be combined with subsequent flatness error evaluation method well. So, we will make an accurate mathematical description of concentric circles in this paper, moreover, considering factors that affect the follow-up methods of evaluation and arranging sample points, we optimize the method of concentric circles to better adapt to our needs to assess the method of error measurement.

\section{ESTABLISHING CONCENTRIC CIRCULAR SELECTION OF SAMPLE POINTS}

\section{A. The Mathematical Description of Concentric Circular Selection of Sample Points}

The method of arranging sample points is discontinuous in the mathematical description, as a result, the parameters are independent of each other. The main parameters of the method are the step of head and circular motion speed of benchmark, we set that the step of head is $r$, the circular speed of benchmark is $\omega$, when the head reaches one position of a step, the benchmark rotate a cycle to take some measure. Because the radius and the number of points may be independently in each circle what concentric circles are composed of, and we can make that the radius of $i+1^{\text {th }}$ circle is more one step $r$ of the head than former. The concentric circular selection of sample points is described as follows in the polar.

$$
L_{i}=i \times \frac{R}{M} \quad i=1,2, \cdots, M ; j=1,2, \cdots, N
$$

$$
\theta_{j}=j \times \frac{2 \pi}{N} \quad i=1,2, \cdots, M ; j=1,2, \cdots, N
$$

Where, $L_{i}$ is the diameter of $j^{\text {th }}$ point of the $i^{\text {th }}$ circle in the polar, $\theta_{j}$ is the angle of $j^{\text {th }}$ point of the $i^{\text {th }}$ circle in the polar, $R$ is the radius of benchmark, $M$ is scores of radius, $N$ is scores of circle. The concentric circular selection of sample points can be described as follows in the rectangular coordinates system.

$$
x_{i}=i \times \frac{R}{M} \times \cos \frac{2 \pi j}{N} \quad i=1,2, \cdots, M ; j=1,2, \cdots, N
$$




$$
y_{j}=i \times \frac{R}{M} \times \sin \frac{2 \pi j}{N} \quad i=1,2, \cdots, M ; j=1,2, \cdots, N
$$

Among, $x_{i}-X$-coordinate of $j^{\text {th }}$ point of the $i^{\text {th }}$ circle, $y_{j}-Y$-coordinate of $j^{\text {th }}$ point of the $i^{\text {th }}$ circle, $R$-the radius of benchmark, $M$-scores of the radius, $N$-scores of the circle.

\section{B. The Explanation of Parameters and the Influence on the} Method of Arranging Sample Points in Mathematical Expression

From the formula (3), (4) we can see, we need to determine three parameters $R, M$ and $N$ in the mathematical description. $R$ is measured parameters of revolving body end-face determined by the radius of measured plane, and It depends on the distance between the maximum radius concentric circle and the actual measuring work-piece according to the measurement standards. The bigger $M$ is, the smaller radial distance of the concentric circles of each round is, and the more dense points cover in the radial, as is shown in Figure $I$.

$N$ has influence on both the position and the distribution of sample points. The bigger $N$ is, the larger the number of points, and the more dense they cover on the circumference, as is shown in Fig.II.

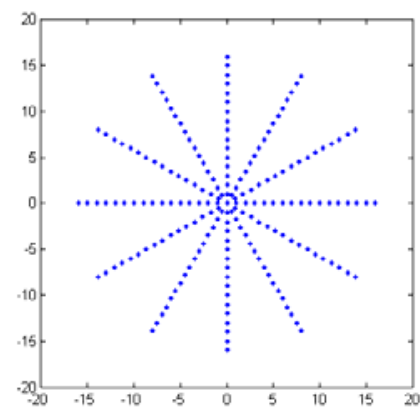

(a) $M=16$, the figure of concentric circular selection of sample points

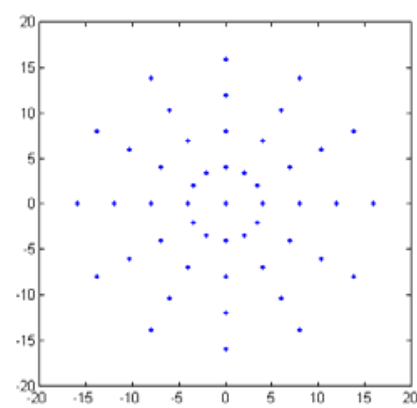

(b) $M=4$, the figure of concentric circular selection of sample points FIGURE I. THE INFLUENCE OF THE RADIUS EQUAL NUMBER M ON BOTH THE LOCATION AND DISTRIBUTION OF THE POINTS

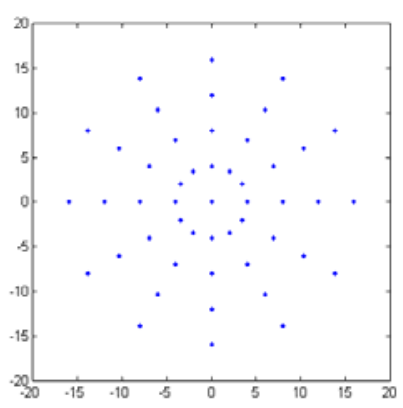

(a) $N=12$, the figure of concentric circular selection of sample points

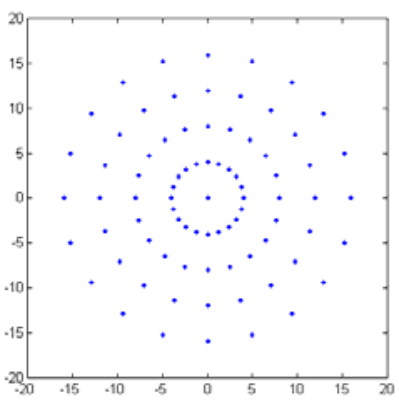

(b) $N=20$, the figure of concentric circular selection of sample points

FIGURE II. THE INFLUENCE OF CIRCUMFERETIAL EQUAL NUMBER N ON BOTH THE LOCATION AND DISTRIBUTION OF THE POINTS

\section{THE OPTIMIZATION OF CONCENTRIC CIRCULAR SELECTION OF SAMPLE POINTS}

Because $M$ and $N$ are two independent parameters, and $M$ has made the radius take the average, so optimizing parameters of concentric circular is mainly to optimize $N$. We can adjust $N$, or give a certain discipline of $N$ to make the location of points change.

From Figure I to Figure II, we can make a conclusion that these points present radially and in a straight line. The points are dense at the begining, however, with the concentric circles of gradually increasing radius, they become fragmented. This does not guarantee an equal distribution of the points, which has negative effect on assessment for least-square method in later. We can design parameters of the method through self-developed algorithm, and regulate $N$ to achieve the desired effect of points by the condition of algorithm. Thus, we can not only make the arbitrariness of arranging sample points to be stronger, but also makes them more average. FigureIIIis the figure of points generated when $N$ with $2 \pi$ cycle adds 2 as $R=36, M=6$, We can see from the graph, although this figure is also concentric circular selection of sample points, but doesn't have very strong regularity of the method, and points appear more uniform. 


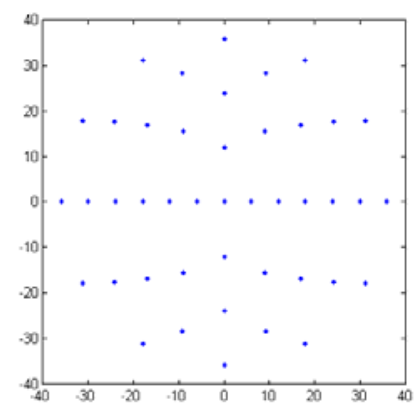

FIGURE III. THE FIGURE OF OPTIMIZED CONCENTRIC CIRCULAR SELECTION OF SAMPLE POINTS

\section{Establishing the MATHEMATiCAL Model of LEAST-SQUARE METHOD OF CONCENTRIC CIRCULAR SELECTION OF SAMPLE POINTS}

If $N$ is always the even number in the process of arranging sample points, then the $x$ and $y$ coordinate system of every discrete sampling point have a symmetrical center. What's more, if the origin $o$ of the space rectangular coordinates system oxyz established in the actual plane is coincident with benchmarks set of symmetrical center, we can undertake Boolean manipulation. At the moment, we can establish mathematical model for the measure of flatness error by using the orthogonal least square method. And the algorithm of concentric circles is greatly simplified than spiral, so as to increase the flatness error evaluation efficiency.

In the space rectangular coordinates system oxyz, the general equation for plane is

$$
A x+B y+C z+D=0
$$

If $a=-A / C, b=-B / C, d=-D / C$, then the general equation for plane can be written as

$$
z=a x+b y+d
$$

In the case of discrete sampling, the least squares plane equation is

$$
\begin{gathered}
\mathscr{q}_{i \rho} \mathrm{\rho}=a x_{i}+b y_{j}+d, \\
i=1,2, \cdots, m ; j=1,2, \cdots, n
\end{gathered}
$$

Type (7), $d$ is the least square plane and $Z$ axis intersection point of $z$ coordinate; $a$ is the least squares plane and $x O z$ coordinate plane intersection slope; $b$ is the least squares plane and yoz coordinate plane intersection slope.
The distance between each sampling point $Q_{i j}\left(x_{i}, y_{j}, z_{i j}\right)$ $(i=1,2, \cdots, m ; j=1,2, \cdots, n)$ and corresponding point in the least squares plane is

$$
\begin{gathered}
\varepsilon_{i j}=\frac{z_{i j}-q_{i j}}{\sqrt{1+a^{2}+b^{2}}}=\frac{z_{i j}-a x_{i}-b y_{j}-d}{\sqrt{1+a^{2}+b^{2}}}, i=1,2, \cdots, m ; \\
j=1,2, \cdots, n
\end{gathered}
$$

But, $a$ and $b$ are small, so there are:

$$
\varepsilon_{i j}=z_{i j}-q_{i \rho}=z_{i j}-a x_{i}-b y_{j}-d, i=1,2, \cdots, m ; j=1,2, \cdots, n
$$

Where, $z_{i j}$ is each discrete sampling points of $z$ coordinate. According to the least square method, there is

$$
\left\{\begin{array}{l}
d=\sum_{i=1}^{m} \sum_{j=1}^{n} z_{i j} / m n \\
a=\sum_{i=1}^{m} \sum_{j=1}^{n} x_{i} z_{i j} / n \sum_{i=1}^{m} x_{i}^{2} \\
b=\sum_{i=1}^{m} \sum_{j=1}^{n} y_{j} z_{i j} / m \sum_{j=1}^{n} y_{j}^{2}
\end{array}\right.
$$

From each sampling point $Q_{i j}\left(x_{i}, y_{j}, z_{i j}\right)(i=1,2, \cdots, m$; $j=1,2, \cdots, n)$ to corresponding point in the least squares plane, the deviation of $z$ coordinate is

$$
\varepsilon_{i j}=z_{i j}-a x_{i}-b y_{j}-d, i=1,2, \cdots, m ; j=1,2, \cdots, n
$$

So, the flatness error from orthogonal least square method

$$
\begin{gathered}
f=\max \left\{\varepsilon_{i j}\right\}-\min \left\{\varepsilon_{i j}\right\} \\
=\max \left\{z_{i j}-a x_{i}-b y_{j}\right\}-\min \left\{z_{i j}-a x_{i}-b y_{j}\right\},
\end{gathered}
$$

$$
i=1,2, \cdots, m ; j=1,2, \cdots, n
$$

\section{THE Simulation OF Flatness ERROR MEASUREMENT}

\section{A. The Method of Acquiring Simulated Data in Computer}

If want to verify the feasibility of a mathematical model, or is the characteristic of mathematical model itself, then we need to import the corresponding data for the calculation. As far as the mathematics model of concentric circular for flatness error measurement is concerned in the paper, it mainly studies the feasibility of mathematical model itself, and gives the 
corresponding results with different parameters of the model. The data used in this paper, is from the thesis published in the high level journal, and is what author M.Affan Badar, Shivakumar Raman obtain through the experiment. The concrete experimental conditions are as follows ${ }^{[3,4]}$ :

We process a $100 \times 100 \mathrm{~mm}$ plane with the method of grinding, and measure the plane by Brown \& Sharpe Reflex 343 series of three coordinates measuring instrument. The article makes the method of arranging sample points to use grids, by the least square method for flatness error measurement, the results of measurement are as follows: The three geometric parameters in ideal plane are $a=-0.00091, b=-0.00067, d=85.0528$, relative to the plane of ideal the highest point error is $e_{\max (+)}=0.06793$, the lowest point error is $e_{\max (-)}=0.06796$, flatness error is $F_{e}=0.13589$.

Considering the method of in the paper is different from above, we should think whether those points can be availed directly. Because the established mathematical model is to apply the least square method, as well as literature[3, 4], so changing the method of arranging sample points has little impact on the least square method to assess, when the length unit of the points is same, and the area is similar.

\section{B. To Determine Concentric Circular Selection of Sample Points and Analyze the Simulation Result}

We put experimental data into the mathematical model to achieve three main parameters in the least square ideal plane equation, $a=0.1544 \times 10^{-3}, b=-0.6148 \times 10^{-4}, d=-84.8742$, then invoke Pingding function to get the results of flatness error measurement $e_{\max (+)}=0.0536, e_{\max (-)}=-0.0835, F_{e}=0.1371$, the highest point is 84th sample point, the lowest point is 13th sample point.

Through the simulation analysis we can see: in the case of concentric circular selection of sample points, flatness error by the least square method differ the traditional pitch method whose results are $0.95 \%$. But we should notice a bit, the simulation is in different method, so we cannot expect that results are completely consistent. While the difference of 0.95 of the results corresponds with the basic conclusion which we mentioned above in error prediction, which can explain that the mathematical model built in the paper can be applied to flatness error measurement.

As we know, both grids and concentric circular selection of sample points for flatness error measurement measure data $Z$. When evaluate concentric circular selection of sample points for flatness error in the paper, we use the orthogonal least square method which is also used by grids. So the coefficient matrix $A$, which the orthogonal least square method assess in the method and the original, is the same in form. Therefore, the main difference is coordinates data produced by the method of arranging sample points between two methods

\section{CONCLUSION}

The paper establish mathematical model of concentric circular selection of sample points for flatness error measurement, it is different from the previous has many restrictions, whose points must spread in the straight line. The points may be set on any position with any quantity in the circumference and radial, which meets the need of flatness error measurement. Because of the central symmetry of concentric circular selection of sample points, we choose the orthogonal least square method, and the calculation of the method and the matrix is simple, which increases the calculation precision.

\section{ACKNOWLEDGEMENT}

This project was supported by National Natural Science Foundation of China in 2015 (No. 51575096).

\section{REFERENCES}

[1] Bi Fei, Yujian Fan, Wenxiong. Xu Adaptive Sampling Method: A New Sampling Method for the Measurement of Geometric Forms. Journal of Applied Sciences, 1994, 12 (4): 1423-430

[2] Taicang Zhang. Research of Arrange Measuring Line and Point in Pitch Measurement of Flatness. Journal of Astronautic Metrology and Measurement, 1993, 12(6): 33-38

[3] M.Affan Badar, Shivakumar Raman, Pakize S.Pulat. Intelligent Search-Based Selection of Sample Points for Straightness and Flatness Estimation [J], Journal of Manufacturing Science and Engineering, 2003, 125: 263-270

[4] M.Affan Badar, Shivakumar Raman, Pakize S.Pulat. Experimental Analysis of Search-Based Selection of Sample points for Straightness and Flatness Estimation [J], Journal of Manufacturing Science and Engineering, 2005, 127: 97-101 